\title{
Efeito do tamanho de gota e volume de aplicação na deposição de agrotóxicos em folhas de videiras ${ }^{1}$
}

\section{Effect of droplet size and spray volume on chemical deposition on grapevine leaves}

\author{
Otavio Jorge Grigoli Abi Saab ${ }^{2 *}$; Ulisses Rocha Antuniassi ${ }^{3}$; \\ Inês Cristina de Batista Fonseca ${ }^{4}$; Werner Genta ${ }^{5}$; Marcelo Batistela ${ }^{6}$
}

\section{Resumo}

A cultura da videira (Vitis vinifera L.) tem se mostrado como alternativa cada vez mais viável dentro da agricultura brasileira, utilizando grande contingente de mão-de-obra, elevado consumo de insumos e adoção de tecnologias atualizadas. Por serem altamente suscetíveis às doenças fúngicas, a variedade Itália e suas mutações somáticas exigem um cuidadoso tratamento fitossanitário, podendo necessitar de mais de 50 pulverizações num único ciclo. O objetivo deste trabalho foi avaliar a influência do tamanho de gota e do volume de aplicação na deposição de calda nas folhas da videira. Os resultados mostraram que o aumento do volume de calda propiciou maior depósito sem, entretanto, alterar o nível de perdas. Não houve interação entre tamanho de gotas e volume de calda, nem influência do tamanho das gotas no depósito de calda nas folhas. O método utilizado mostrou-se adequado aos objetivos deste trabalho.

Palavras-Chave: Vitis Vinifera L., agrotóxicos, pulverização

\begin{abstract}
Grapevines (Vitis vinifera L.) are a good option for Brazilian growers using large amount of labor, resources and technology. Itália variety and its mutations require careful treatments since they are very prone to develop diseases. The aim of this study was evaluate the influence of droplet size and spray volume in the chemical deposition on grapevine leaves. The increase of the spray volume provided better deposition. There was no interaction between droplet size and spray volume as well as there was no influence of droplet size on the spray deposits on the leaves. The method can be considered adequate for the propose of this work.
\end{abstract}

Key words: Vitis Vinifera L., chemicals, sprayer

\footnotetext{
1 Trabalho realizado em Marialva, PR.

2 Engenheiro Agrônomo, Doutor, Professor Adjunto, UEL/CCA/Agronomia. E-mail: abisaab@uel.br.

3 Professor Adjunto, Dep. de Engenharia Rural, FCA/UNESP, Botucatu, SP.

4 Professora Adjunta, UEL/CCA/Agronomia, Londrina, PR.

5 Eng. Agrônomo, PLANTA, Marialva, PR.

${ }^{6}$ Discente de agronomia, UEL/CCA/Agronomia, Londrina/PR.

* Autor para correspondência.
} 


\section{Introdução}

As variedades de uva consideradas 'finas de mesa', Itália e suas mutações somáticas, são muito sensíveis ao ataque de doenças fúngicas. A associação de fatores como suscetibilidade à doença, condições climáticas favoráveis e forma de condução obriga o viticultor a realização de até 50 pulverizações em um único ciclo da cultura, que dura aproximadamente 150 dias. A região norte do Estado do Paraná é responsável por aproximadamente $20 \%$ do total da área cultivada com uvas finas de mesa no Brasil, sendo que só o município de Marialva representa mais de $10 \%$.

Genta (2000) afirma que o sistema de condução mais difundido na região é a latada (caramanchão), sendo que este tipo de estrutura é capaz de sustentar a produção, contribuindo para o aumento da produtividade e facilitando os tratos culturais, fitossanitários e a colheita. A maior parte dos viticultores utiliza sistemas pulverizadores semi-estacionários desenvolvidos no local para aplicação dos agrotóxicos. Esses sistemas são compostos de reservatório, bomba de pistões, mangueiras e lança com bicos de pulverização. A aplicação é feita manualmente de baixo para cima, conforme descrito por Abi Saab (1996), e representada na Figura 1.

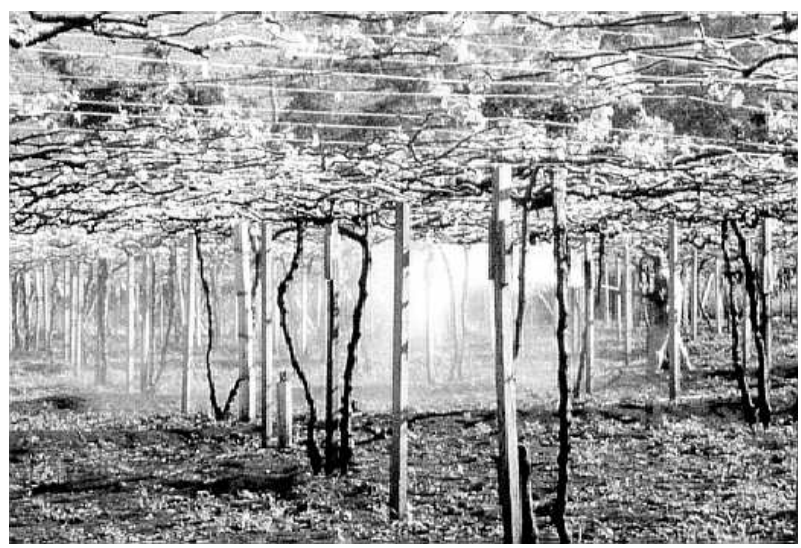

Figura 1 - Detalhe de aplicação manual com o equipamento semi-estacionário.

Comparações são necessárias para que surjam melhorias na segurança e efetividade dos métodos de aplicação de agrotóxicos nas plantas (CROSS et al., 1997). A tendência natural é testar a eficácia dos agrotóxicos diretamente em experimentos de campo, porém experimentos desse tipo são demorados e o sucesso está sujeito à ocorrência de pragas e/ ou doenças em intensidade adequada e a variações do clima. Além disso, tais experimentos podem ser incongruentes devido à variabilidade biológica, e pode ser que tenham de ser repetidos em vários locais e estações, para expor diferenças entre tratamentos. Cross et al. (1997) consideraram que, na avaliação da efetividade dos métodos de aplicação, a quantificação dos depósitos de pulverização constituiu-se em um dos meios mais rápidos e menos exigentes de recursos. Muitos são os métodos utilizados para a avaliação da deposição da calda, tais como a colorimetria (SALYANI; WHITNEY, 1988; OZMERI; CILINGER, 1992), a espectrometria (DERKSEN; GRAY, 1995; PLANAS et al., 1996; HALL; WESTERN; HOLLOWAY., 1997; GIL et al. 1998; PLANAS; SOLONELLES; FILLAT, 2000; GIL, 2000) e a fluorimetria (NORBDO, 1992; PERMIN; JORGERSEN; PERSSON，1992; PERGHER; GUBIANI, 1996; NILSSON, 1998). O tamanho das gotas e/ou o volume de aplicação da calda, e sua relação com a deposição, é uma constante em muitos trabalhos. Steden (1992) argumenta que a importância do tamanho das gotas aumenta em função do aumento da dificuldade de alcance do alvo; para a condição estudada, gotas menores proporcionaram melhores resultados. Neste mesmo trabalho, o autor faz outras considerações importantes. Citando que, quanto ao volume de calda aplicado para aplicações de fungicidas em videiras, foram obtidos bons resultados para aplicações com volumes mais baixos (150 a $500 \mathrm{~L} / \mathrm{ha}$ ). Volumes maiores proporcionaram grandes perdas por escorrimento nas diversas partes da planta. A condutividade elétrica de soluções como parâmetro de avaliação foi utilizada por Velini e Antuniassi (1992), visando a determinação de vazões em sistemas de pulverização. Este processo apresenta grande versatilidade e precisão para a quantificação da calda depositada, que 
é determinada em função da condutividade elétrica da solução de lavagem de alvos (VELINI et al., 1995). Permite também, em muitos casos, a utilização da própria folha das plantas para a amostragem, conforme exemplificado no trabalho de Abi Saab (1996). Isso é importante porque nenhum alvo artificial representa melhor a folha que ela mesma. Outros exemplos da utilização deste conceito pode ser observado nos trabalhos de Nogueira (1996), Antuniassi, Velini e Martins (1996) e Fox et al. (1998). A determinação das perdas, entendida como a parcela do produto não depositada no local desejado da planta, é uma avaliação importante dos sistemas de pulverização, sendo comumente utilizada. Os trabalhos apresentados por Val et al. (1988), Irla (1990) e Permin, Jorgersen e Persson (1992) podem ser citados como exemplos. Também é comum a associação da avaliação da deposição com a avaliação das perdas. Os trabalhos de Abi Saab (1996) e Holownicki et al. (2000) são exemplos destas avaliações conjuntas. O objetivo deste trabalho foi avaliar a influência da variação do tamanho da gota e do volume aplicado na deposição da calda aplicada nas folhas de videiras.

\section{Material e Métodos}

O trabalho foi desenvolvido na propriedade denominada Sítio Inumaru, estrada Sarandi km 10 (2324'17''S e 5148'19''W), situada no município de Marialva-PR. A região tem altitude aproximada de $600 \mathrm{~m}$. A classificação climática, segundo W. Köeppen, é Cfa. A parreira utilizada, da variedade Rubi, tinha aproximadamente 4 anos, plantada em alinhamento alternado com espaçamento de $3 \mathrm{~m}$ entre linhas e $9 \mathrm{~m}$ entre plantas, com área aproximada de $5.700 \mathrm{~m}^{2}$. A forma de condução utilizada é em latada, com altura aproximada de $2 \mathrm{~m}$. A parreira estava com condução normal de produção, já apresentando cachos.

Para simular as aplicações feitas com o equipamento do viticultor, usualmente um equipamento semi-estacionário, adaptou-se uma mangueira de aproximadamente $100 \mathrm{~m}$, provida de registro, manômetro e lança com 2 bicos de pulverização a um pulverizador marca KO modelo A500, tracionado e acionado por um trator Agrale 4100. As pontas de pulverização utilizadas foram D3 Yamaho e 110-LD02 Jacto, ambas de jato plano.

Para a realização das atividades deste trabalho utilizaram-se os seguintes equipamentos e materiais diversos: manômetro padrão, com precisão de $0,5 \%$ e escala de 0 a $1600 \mathrm{kPa}$, manômetro de serviço com escala de 0 a $2100 \mathrm{kPa}$, condutivímetro digital, equipado com célula termocompensadora e escala variável de 0 a $20.000 \mathrm{mS}$, termohigrômetro, corante fluorescente derivado de uréia/formaldeído (Luxcor LRM 100), cloreto de potássio granulado para adubação, fungicida cymoxanil + maneb (Curzate M + Zinco), água destilada e deionizada, baldes plásticos, sacos plásticos, trena, réguas plásticas, barbante, calculadoras, balanças, dispensador, micro-pipetas e vidraria em geral.

Para a avaliação da deposição, o método para obtenção da calda utilizada foi o mesmo empregado por Abi Saab (1996), sendo composta da mistura do fungicida, cloreto de potássio e corante fluorescente em água, nas seguintes proporções: $100 \mathrm{~L}$ de água, $200 \mathrm{~g}$ de fungicida (produto comercial), $5.000 \mathrm{~g}$ de cloreto de potássio e $715 \mathrm{~g}$ de corante.

Para conhecer os tamanhos das gotas geradas pelos bicos utilizados, realizou-se a medição através de um equipamento analisador de partículas por difração de laser marca Malvern, modelo Mastersizer S long bed versão 2.15, utilizando lente de $300 \mathrm{~mm}$ de distância focal, disponibilizado pelo Departamento de Defesa Fitossanitária da FCAV/UNESP/ Jaboticabal.

O experimento foi conduzido em blocos ao acaso em esquema fatorial $2 \times 2$ (volume de calda $\mathrm{x}$ tamanho de gota) e 5 repetições, em parcelas de 12,5 $\mathrm{m}^{2}(2,5 \times 5 \mathrm{~m})$. Após as aplicações, os valores médios de volume de calda (L/ha), calculados em função da vazão e do tempo em cada parcela, foram determinados e estão descritos na Tabela 1 . 
Saab, O. J. G. A. et al.

Tabela 1 - Caracterização dos tratamentos utilizados.

\begin{tabular}{ccccccc}
\hline Tratamento & $\begin{array}{c}\text { Tipo de } \\
\text { Ponta }\end{array}$ & $\begin{array}{c}\text { Pressão } \\
(\mathrm{kPa})\end{array}$ & $\begin{array}{c}\text { Vazão/bico } \\
(\mathrm{L} / \mathrm{min})\end{array}$ & $\begin{array}{c}\text { Tempo médio } \\
\left(\mathrm{s} / 12,5 \mathrm{~m}^{2}\right)\end{array}$ & $\begin{array}{c}\text { Volume médio } \\
\text { calda }(\mathrm{L} / \mathrm{ha})\end{array}$ & $\begin{array}{c}\text { DMV } \\
(\mu \mathrm{m})\end{array}$ \\
\hline 1 & D3 & 1050 & 0,7 & 17,0 & $312(\mathrm{VB})$ & $57(\mathrm{GP})$ \\
2 & $110-\mathrm{LD}-02$ & 210 & 0,7 & 16,4 & $307(\mathrm{VB})$ & $205(\mathrm{GG})^{*}$ \\
3 & D3 & 1050 & 0,7 & 30,2 & $567(\mathrm{VA})$ & $57(\mathrm{GP})$ \\
4 & $110-\mathrm{LD}-02$ & 210 & 0,7 & 30,6 & $569(\mathrm{VA})$ & $205(\mathrm{GG})^{*}$ \\
\hline
\end{tabular}

GP e GG: gotas menores e maiores, respectivamente; VB e VA: volumes baixo e alto, respectivamente.* = dado fornecido pelo fabricante, obtido no mesmo tipo de equipamento; DMV = diâmetro mediano volumétrico

As parcelas foram protegidas por uma cortina plástica, assim como utilizado por Abi Saab (1996) (Figura 2), para evitar que a deriva durante as aplicações contaminasse as parcelas vizinhas. As aplicações foram executadas sempre pela mesma pessoa (o encarregado deste serviço na propriedade), objetivando manter as características normais de trabalho. Os bicos foram mantidos a uma distância aproximada de $50 \mathrm{~cm}$ das folhas e, antes de cada aplicação, a vazão de calda foi aferida coletando-se o volume de líquido oriundo da lança de pulverização em um saco plástico, durante um tempo determinado. A calda coletada foi transferida para uma proveta, aferindo-se o volume e, quando necessário, novas regulagens da pressão foram realizadas, até se atingir a vazão pretendida.

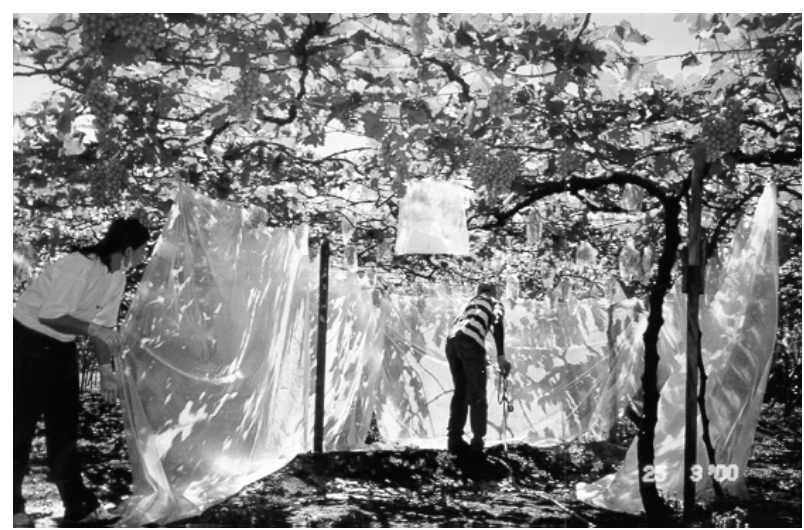

Figura 2 - Aplicação na parcela protegida por cortina plástica.

Para a avaliação das perdas e do depósito volumétrico da calda sobre as folhas, utilizou-se o método baseado na determinação da condutividade elétrica da solução pulverizada, sugerida por Velini et al. (1995) e descrita por Abi Saab (1996).
Utilizou-se a equação a seguir, proposta por Abi Saab (1996), para correlacionar a área foliar com o resultado da multiplicação do comprimento pela largura da folha:

$$
\mathrm{Af}=0,920897 \times \mathrm{Cf} \times \mathrm{Lf}-\left(\mathrm{r}^{2}=0,97\right),
$$

onde:

Af $=$ área estimada da folha $\left(\mathrm{cm}^{2}\right)$

$\mathrm{Cf}=$ comprimento da folha, medido da inserção do pecíolo até a extremidade $(\mathrm{cm})$.

$\mathrm{Lf}=$ largura máxima da folha, medida entre as extremidades laterais $(\mathrm{cm})$.

Utilizando-se de micro-pipetas e buretas, volumes conhecidos da calda foram depositados sobre folhas retiradas das plantas. Após a deposição, cada folha foi colocada em um saco plástico, onde foram introduzidos $30 \mathrm{ml}$ de água destilada e deionizada. O conjunto folha, saco plástico e água foi agitado durante 30 segundos, e imediatamente após foi medida a condutividade elétrica da solução obtida. A seguir, foram determinados o comprimento e a largura máxima das folhas, através de medição com régua plástica, para estimativa da área de cada folha. Nas mesmas condições, procedeu-se também a avaliação da condutividade elétrica da solução de lavagem de folhas sem a calda e de sacos plásticos sem as folhas, com o objetivo de complementar os dados necessários para a elaboração da equação.

De posse destes dados, determinou-se uma nova equação correlacionando volume de calda deposita- 
do sobre as folhas, condutividade da solução de lavagem e área foliar.

$\mathrm{Dtf}=0,409003 \times \mathrm{Cs}-0,14881 \times$ Af $-16,4347-\left(\mathrm{r}^{2}=0,99\right)$,

onde:

$\mathrm{Dtf}=$ depósito total de calda estimado sobre a folha $(\mathrm{mL})$ $\mathrm{Cs}=$ condutividade elétrica da solução de lavagem da folha $(\mathrm{mS} / \mathrm{cm})$

Af = área estimada da folha $\left(\mathrm{cm}^{2}\right)$

Após aproximadamente 15 minutos da aplicação de cada tratamento, tempo suficiente para a secagem da calda, foram coletadas, ao acaso, 30 folhas de cada parcela, sendo imediatamente colocadas, individualmente, em sacos plásticos com identificação. Estas folhas foram submetidas aos processos de lavagem, estimativa da área foliar e determinação da condutividade elétrica das soluções obtidas.

Os dados de condutividade foram aplicados à Equação 2, fornecendo valores estimados do volume total de calda depositado em cada folha. Os valores obtidos nesta equação foram aplicados à Equação 3, fornecendo o depósito estimado de calda por $\mathrm{cm}^{2}$ de folha.

$$
\mathrm{Vcf}=\mathrm{Dtf} / \mathrm{Af}
$$

onde:

$\mathrm{Vcf}=$ volume de calda depositado sobre a folha $\left(\mathrm{mL} / \mathrm{cm}^{2}\right)$ $\mathrm{Dtf}=$ depósito total de calda estimado sobre a folha $(\mathrm{mL})$ Af $=$ área estimada da folha $\left(\mathrm{cm}^{2}\right)$

Em cada tratamento a estimativa das perdas foi realizada através da comparação entre o volume de calda recuperado nas folhas e o volume efetivamente aplicado durante as pulverizações. Para a determinação do volume recuperado realizou-se uma avaliação da área foliar correspondente a $1 \mathrm{~m}^{2}$ de solo na cultura (índice de área foliar). Assim, multiplicando-se o depósito médio pela área foliar obteve-se o total de de- pósito correspondente a $1 \mathrm{~m}^{2}$ de solo. Comparandose este valor com o volume de calda efetivamente aplicado, obteve-se as perdas estimadas.

As análises estatísticas foram feitas através da Análise de Variância complementada com o teste de Tukey para comparação de médias, utilizando-se o programa $\operatorname{SAS}^{\circledR}$ (1996).

\section{Resultados e Discussão}

As Tabelas 2 e 3 apresentam os resultados relativos ao depósito de calda nas folhas e respectivas perdas.

Tabela 2 - Análise de variância do depósito de calda recuperado nas folhas $\left(\mathrm{mL} / \mathrm{cm}^{2}\right)$, com respectivas médias, dos fatores gota e volume, e da interação entre eles.

\begin{tabular}{|c|c|c|c|}
\hline \multicolumn{3}{|l|}{ Fator } & Valor de F \\
\hline \multicolumn{3}{|c|}{ Tamanho das Gotas } & $0,27 \mathrm{n} . \mathrm{s}$ \\
\hline \multicolumn{3}{|c|}{ Volume de Calda } & $50,67 * *$ \\
\hline \multicolumn{3}{|c|}{ Gotas x Volumes } & 0,01 n.s. \\
\hline \multicolumn{3}{|l|}{ Blocos } & $13,56 * *$ \\
\hline \multicolumn{3}{|l|}{ C.V. $(\%)$} & 66,72 \\
\hline Tratamentos & Gotas & Volumes & Médias \\
\hline 1 & GP & VB & 0,74 \\
\hline 2 & GG & VB & 0,77 \\
\hline 3 & GP & VA & 1,12 \\
\hline 4 & GG & VA & 1,14 \\
\hline \multicolumn{4}{|c|}{ Médias para volumes } \\
\hline \multicolumn{3}{|c|}{ VB } & $1,13 \mathrm{a}$ \\
\hline \multicolumn{3}{|c|}{ VA } & $0,75 \mathrm{~b}$ \\
\hline \multicolumn{4}{|c|}{ Médias para gotas } \\
\hline \multicolumn{3}{|c|}{ GG } & $0,95 \mathrm{a}$ \\
\hline \multicolumn{3}{|c|}{ GP } & $0,93 \mathrm{a}$ \\
\hline
\end{tabular}

GP e GG: gotas menores e maiores, respectivamente; VB e VA: volumes baixo e alto, respectivamente. As médias seguidas de mesma letra não diferem pelo teste de Tukey no nível de $5 \%$ de probabilidade. $* *$ indica significância no nível de $1 \%$ de probabilidade. n.s. indica que não houve significância. 
Observa-se que na análise dos depósitos de calda nas folhas não houve influência do tamanho das gotas. Entretanto, o volume maior propiciou maior depósito, com diferença estatisticamente significativa, concordando com os resultados apresentados no trabalho de Salyani e Whitney (1988). Não houve interação significativa entre tamanho das gotas e volume de calda. Os volumes de aplicação utilizados (entre 300 e $600 \mathrm{~L} / \mathrm{ha}$ ) são próximos dos valores considerados como de melhor desempenho por Steden (1992), ou seja, representam volumes de calda com menor possibilidade de escorrimento.

Analisando-se do ponto de vista da eficiência biológica do agrotóxico, na hipótese de se considerar importante a quantidade depositada na folha (ação sistêmica), independentemente da cobertura, reforça-se o conceito de que as gotas maiores devem ser preferidas em função do menor potencial de ocorrência de deriva, já que não houve diferença estatística significativa nos depósitos quando do uso de gotas grandes ou pequenas.

Tabela 3 - Análise de variância, com respectivas médias, da porcentagem de perdas da calda.

\begin{tabular}{lccc}
\hline Fator & & \multicolumn{2}{c}{ Valor de F } \\
\hline Tratamentos & & & 1,48 n.s. \\
Blocos & & & 0,58 n.s. \\
C.V. (\%) & & & 12,19 \\
\hline Tratamentos & Gotas & Volumes & Médias \\
\hline 1 & GP & VB & $64,52 \mathrm{a}$ \\
2 & GG & VB & $63,24 \mathrm{a}$ \\
3 & GP & VA & $70,58 \mathrm{a}$ \\
4 & GG & VA & $69,67 \mathrm{a}$ \\
& & &
\end{tabular}

GP e GG: gotas menores e maiores, respectivamente; VB e VA: volumes baixo e alto, respectivamente. As médias seguidas de mesma letra não diferem entre si pelo teste de Tukey no nível de $5 \%$ de probabilidade. n.s. indica que não houve significância.
É importante ressaltar que a utilização de maiores volumes promoveu melhores resultados. Entretanto, os maiores valores de volume utilizados neste trabalho são, em geral, equivalentes aos menores volumes aplicados na prática pelos agricultores.

\section{Conclusões}

Para as condições específicas deste trabalho podemos concluir que:

1. O aumento do volume de calda propiciou melhor depósito sem, entretanto, alterar o nível percentual de perdas;

2. A deposição de calda nas folhas independe do tamanho das gotas e da interação com volume de calda;

3. O método utilizado mostrou-se adequado aos objetivos deste trabalho.

\section{Agradecimentos}

Nossos agradecimentos à ANPEF - Associação Norte Paranaense de Estudos em Fruticultura e à Máquinas Agrícolas JACTO S.A.

\section{Referências}

ABI SAAB, O. J. G. Avaliação de um sistema de aplicação de defensivos utilizado em videiras no Município de Londrina/PR. 1996. 65f. Dissertação (Mestrado em Agronomia) - Faculdade de Ciências Agronômicas, Universidade Estadual Paulista, Botucatu.

ANTUNIASSI, U. R.; VELINI, E. D.; MARTINS, D. Spray deposition and drift evaluation of air-carrier peach orchard sprayers. AgEng, Madrid, p.96-136, 1996.

CROSS, J. V. et al. Quantification of spray deposits and their variability on apple trees. In.: ASPECTS OF APPLIED BIOLOGY, 48., 1997, Long Ashton. Proceedings... Warwick : Association of Applied Bilogists, 1997. p.217-24.

DERKSEN, R. C.; GRAY, R. L. Deposition and air speed patterns of air-carrier apple orchard sprayer. Transactions of the Asae, St. Joseph, v.38, n.1, p.5-11, 1995. 
FOX, R. D. et al. The reichard spray deposit analyser, a tool for measuring spray deposit by measuring condutivity. AgEng, Oslo, n.98, p.1-7, 1998.

GENTA, W. A cultura da Videira. Marialva: PLANTAPlanejamento e Assistencia Técnica, 2000.

GIL, E. et al. Improvement of the pesticide applications in vineyard. Relationship between methodology of application and quality parameters. AgEng, Oslo, n. 98 p.A-015, 1998.

GIL, E. Characterisation of two different conceptions of pneumatic sprayer in vineyard applications. AgEng., Warwick, n. 00, p.PM-033, 2000..

HALL, K. J.; WESTERN, N. M.; HOLLOWAY, P. J. Effects of adjuvant oil emulsions on foliar retention and spray quality. In.: THE 1997 BRIGHTON CROP PROTECTION CONFERENCE, 1997., Brighton. Proceedings... Brighthon: The British Crop Protection Council, 1997. p.549-54.

HOLOWNICKI, R. et al. Automatic self adjusting airjet sprayer concept for fruit trees. AgEng., Warwick, n.00, p.PM-053, 2000.

IRLA, E. Essais comparatifs de pulvérizateurs pour la vigne, 1989. Tanikon: Rapports FAT, 1990.

NILSSON, U. Spray application and working environment in greenhouse. AgEng., Oslo, n. 98, p.A016, 1998.

NOGUEIRA, H.C. Avaliação da uniformidade de deposição e perdas de calda na utilização de um pulverizador desenvolvido para aplicação de herbicidas em ferrovias. 1996. 72f. Dissertação (Mestrado em Agronomia) - Faculdade de Ciências Agronômicas, Universidade Estadual Paulista, Botucatu.

NORBDO, E. Effects od nozzles size, travel speed and air assistance on deposition on artificial vertical and horizontal targets in laboratory experiment. Crop Protection, Kidlington, v.11, n.3, p.272-8, 1992.

OZMERI, A.; CILINGIR, I. Use of colorimetric technique in determining surface coverage in spraying. Agricultural. Mechanization In Asia, Tokio, v.23, n.1, p.37-8, 1992.

PERGHER, G.; GUBIANI, R. Methods for the assessment of vertical spray distributions from air-assisted sprayers: a comparative study. AgEng., Madrid, n. 96, p.A-127, 1996.
PERMIN, O.; JORGERSEN, L. N.; PERSSON, K. Biological effect of herbicides and fungicides, deposition and drift hazards, when using different sizes of hydraulic flat fan nozzle for the application. Tidsskrift for Planteavl, Lyngby, v.96, n.5, p.531-42, 1992.

PLANAS, S. et al. A proposal of methodology for air assisted sprayers assessment in apple orchards. AgEng., Madrid, n. 96, p.A-149, 1996.

PLANAS, S.; SOLANELlES, F.; FILLAT, A. Challenges of recycling tunnel sprayers (RTS) in mediterranean vineyards and fruit orchards. AgEng., Warwick, n. 00, p.PM-057, 2000. 8p.

SALYANI, M.; WHITNEY, J. D. Evaluation of methodologies for field studies of spray deposition. Transactions of the Asae, St. Joseph, v.31, n.2, p.390-5, 1988.

STEDEN, C. Untersuchungen zum einflub der tropfengröbe auf die belagsbildung und die biologische wirksamkeit gegen Oidium tuckeri Berk. an reben. 1992. $118 \mathrm{p}$. Inaugural Dissertation (Erlangung des Doktorgrades Justus-Liebig)-Universität Gieben, JustusLiebig-Universität, Gieben.

VAL, L. M. et al. Penetracion e tamaño de gota en hoja de distintos sistemas de distribucion de productos fitosanitarios en cultivos de citricos. In.: CONFERENCIA INTERNACIONAL DE MECANIZACION AGRARIA, 20., 1988, Zaragoza. Proceedings... Zaragoza: Asociacion Nacional de Ingenieros Agronomos, 1988. p.201-207.

VELINI, E. D.; ANTUNIASSI, U. R. Desenvolvimento de um sistema para injeção de defensivos agrícolas na barra de aplicação. In: CONGRESSO BRASILEIRO DE ENGENHARIA AGRÍCOLA, 21., 1992, Santa Maria. Anais...Santa Maria: Universidade Federal de Santa Maria, 1992. p.2035-2047.

VELINI, E. D. et al. Utilização da condutividade elétrica para avaliação do depósito da calda de pulverização em alvos ou folhas. In.: CONGRESSO BRASILEIRO DA CIÊNCIA DAS PLANTAS DANINHAS, 20., 1995, Florianópolis. Anais... Florianópolis: Sociedade Brasileira da Ciência das Plantas Daninhas, 1995. p.427. 
